\author{
G. M. Fatterpekar \\ S. K. Mukherji \\ P. Rajgopalan \\ Y. Lin \\ M. Castillo
}

\section{Normal age-related signal change in the laryngeal cartilages}

Received: 12 July 2003

Accepted: 1 December 2003

Published online: 30 June 2004

(C) Springer-Verlag 2004

G. M. Fatterpekar

Department of Radiology, Mt Sinai

Medical Center, New York, USA

S. K. Mukherji $(\bowtie)$

Section of Neuroradiology, Department of Radiology, University of Michigan, 1500 E Medical Center Drive, Ann Arbor MI 48109, USA

E-mail: Mukherji@med.umich.edu

Tel.: + 1-734-9358865

Fax: + 1-734-7642412

P. Rajgopalan $\cdot$ Y. Lin $\cdot$ M. Castillo

Department of Radiology,

University of North Carolina,

Chapel Hill, North Carolina, USA

\begin{abstract}
MRI may be used for detecting cartilage invasion in patients with laryngeal carcinoma. However, the normal laryngeal ossification pattern has not been studied. Our purpose was to examine the normal age-related signal patterns in the cricoid, thyroid and arytenoid cartilages on T1-weighted images. Signal in the cartilages was assessed by two radiologists in a blinded fashion using three-point scales for intensity and symmetry. Statistical analysis consisted of logistic and monotonic regression. There was excellent interobserver agreement $(>85 \%$ ) for all categories. The cartilages predominantly
\end{abstract}

ossify symmetrically and the extent of high signal from all three increases with age. The latter may help in detection of cartilage invasion by tumor in older patients. Normal symmetry may be helpful when comparing sides for tumor invasion.

Keywords Laryngeal cartilages · Ossification · Magnetic resonance imaging

\section{Introduction}

Cartilage invasion by laryngeal cancer has a poor prognosis [1]. Exclusion of cartilage invasion is therefore important as its presence often precludes laryngeal conservation surgery. Cartilage invasion can be investigated using CT or MRI [2-7].

Laryngeal cartilages exhibit a variation in signal characteristics on T1-weighted images depending on their ossification. The fatty marrow in ossified cartilage is identified as an area of high signal with a low-signal rim [8]. Unossified cartilage, with its red marrow is isointense or gives low signal. Laryngeal tumor has signal characteristics similar to unossified cartilage, so that it may be difficult to distinguish normal unossified cartilage from that invaded by tumor. Knowledge of the agerelated changes in the signal characteristics of the laryngeal cartilages may help to improve detection of tumor invasion. Our purpose was to explore the normal age-related signal patterns in the thyroid, cricoid, and arytenoid cartilages.

\section{Materials and methods}

We prospectively studied 60 patients ( 34 females and 26 males aged 2 months to 77 years, average 43 years who underwent MRI studies for cervical radiculopathy. None had a history of hoarseness or laryngeal trauma.

Imaging consisted of contiguous 3-mm T1-weighted images (TR 700 TE $15 \mathrm{~ms}$, one excitation, from the hyoid bone to the base of the cricoid cartilage. The images were assessed for the signal from the laryngeal cartilages by two neuroradiologists blinded to the age of the patients. They used the following scale to score the ossification of the thyroid, cricoid and arytenoid carti- 
lages: 1: no increased signal; 2: mild heterogeneous signal, and 3: homogeneously increased signal compared with that of the cervical strap muscles. A separate rating was also used to describe the right/left symmetry of signal: symmetric or asymmetric.

Statistical analysis was performed by dividing the patients into four age groups: $\leq 25,26-53,54-64$ and 65-77 years. Logistic and reduced monotonic regression were used to examine the effect of age on signal in the three cartilages. Logistic regression relies on assumption of a fairly consistent monotonic trend of the response variable (signal) with respect to the explanatory variable (age). Reduced monotonic regression models the trend as flat, with monotonic jumps at various ages, which are determined from the data. The relationship between age and symmetry was also studied. Wilcoxon's rank sum tests were used to test for age differences for each of the three cartilages.

\section{Results}

A summary of the results is presented in Tables 1 and 2 . The signal demonstrated significant age-related changes in the arytenoid and cricoid $(P<0.0001)$, and thyroid $(P=0.05)$ cartilages, using logistic regression. Reduced monotonic regression categorized age into two groups for all these cartilages with a cut-off at 26 years. Reapplying logistic regression to the 53 patients 26 years of age or more, significant age-related changes were seen for arytenoid cartilages $(P=0.006)$, but not for thyroid $(P=0.75)$ or cricoid cartilages $(P=0.08)$. Table 1 shows the mean scores for the age groups, where 54 years was the age at which reduced monotonic regression identified a second jump in the arytenoid cartilage score. Age 65 years is where the strongest (though not statistically significant) age-related change occurred for the cricoid cartilage.

An increase in the symmetry of the signal was noted for thyroid $(P=0.04)$, but not for arytenoid $(P=0.73)$ or cricoid $(P=0.12)$ cartilages. Most patients had symmetric signal (Table 2).

\section{Discussion}

The laryngeal skeleton consists of two types of cartilage. The epiglottic cartilage consists of elastic cartilage and does not ossify, while the cricoid, thyroid and arytenoid cartilages consist of hyaline cartilage, which demonstrates a predictable pattern of ossification [9]. MRI has been used to examine the larynx $[10,11]$. As age advances, there is a progressive increase in the amount of fatty marrow within the ossified hyaline cartilage. This is seen as an increase in the signal on T1-weighted images [8, 12]. Histologic studies have demonstrated a predictable pattern of ossification in each of the hyaline laryngeal cartilages $[13,14,15]$.

The first evidence of mineralization and osteogenesis is generally seen late in the second decade or early in the third; ossification is rare in the first. Russo et al. [16] demonstrated a rare instance of ossification in the thyroid cartilage in the first year of life. In our series, the earliest evidence of an increase in the signal of the thyroid cartilage relative to the strap muscles was seen in an 8-month-old child.

We noted an increase in signal from the thyroid cartilage with increasing age. The first evidence of increased signal characteristics was seen at 26 years, with a second jump at 54 years (Table 1). A mild drop in signal was seen at 65 years of age, the cause for which could not be ascertained. We suspected an unpredictable or variable pattern of ossification to be responsible. A major drawback of this study has been the small number of patients in this particular age-group. A larger group should provide a more definite pattern of signal characteristics at this age. Most patients showed a symmetrical signal from of the right and left sides of the thyroid cartilages (Table 2), which correlates with previous histologic studies $[14,15]$.

Ossification in the cricoid cartilage usually begins at the same time as in the thyroid [14, 15]. It is first seen in the curvilinear superior border of the lamina and extends along the muscle attachments. We noted a progressive increase in the extent of high signal with increasing age. The two jumps for an increase in signal from the cricoid cartilage were at the 26-53 and 65-77 year age groups (Table 1). The anterior arch of the cricoid cartilage did not show any increase in signal, despite the rest of the cartilage doing so. Histologic studies have demonstrated that the anterior arch is the last part to ossify [14]. The age at which it does so has, however, not been documented. We noted a remarkable degree of symmetry in the signal from the cartilage (Table 2), which also correlates with previous histologic studies $[15,17]$.
Table 1 Mean signal intensity ( \pm s.d.) of laryngeal cartilages on T1-weighted images in 60 patients. 1: no increased signal 2: heterogeneous signal 3: homogeneously increased signal compared with strap muscles

\begin{tabular}{|c|c|c|c|c|}
\hline \multirow[t]{2}{*}{ Cartilage } & \multicolumn{4}{|c|}{ Age (years) and number of patients } \\
\hline & $\leq 25(7)$ & $26-53(41)$ & $54-64(6)$ & $65-77(6)$ \\
\hline Thyroid & $1.4 \pm 0.5$ & $2.3 \pm 0.5$ & $2.7 \pm 0.8$ & $2.5 \pm 0.5$ \\
\hline Cricoid & $1.0 \pm 0.0$ & $2.3 \pm 0.6$ & $2.3 \pm 0.5$ & $2.8 \pm 0.4$ \\
\hline Arytenoid & $1.3 \pm 0.5$ & $2.3 \pm 0.7$ & $2.8 \pm 0.4$ & $2.8 \pm 0.4$ \\
\hline
\end{tabular}


Table 2 Patients (\%) with symmetric signal intensity

\begin{tabular}{lllll}
\hline Cartilage & \multicolumn{4}{l}{ Age (years) and number of patients } \\
\cline { 2 - 5 } & $\leq 25(7)$ & $26-53(41)$ & $54-64(6)$ & $65-77(6)$ \\
\hline Thyroid & $5(71)$ & $32(78)$ & $6(100)$ & $6(100)$ \\
Cricoid & $7(100)$ & $38(93)$ & $6(100)$ & $6(100)$ \\
Arytenoid & $7(100)$ & $35(85)$ & $6(100)$ & $5(83)$ \\
\hline
\end{tabular}

The earliest evidence of ossification of the arytenoid cartilages on histologic studies is seen in the late second or early third decade. Our youngest patient was a 27 year-old man, in whom ossification was also present in the thyroid and cricoid cartilages. A statistically significant increase in the signal characteristics of the arytenoid cartilages involving the apices, body and muscular processes was observed with increasing age. No increase in signal was seen in the vocal processes. Histologic studies have demonstrated the vocal processes to consist of elastic cartilage and therefore failing to ossify $[15,18]$. A remarkable degree of symmetry in the signal was evident (Table 2), as in previous histologic studies $[14,15]$.

Laryngeal carcinomas preferentially invade ossified cartilage [18]. This susceptibility has been attributed to vascularization and to a tumor angiogenetic factor; unossified cartilage is thought to be resistant to tumor infiltration as a result of its capacity to release proteins that inhibit collagenases and tumor angiogenetic factor. The low-signal rim surrounding ossified cartilage on T1-weighted images is due to a chemical shift artifact that results from mismapping of signal at fat/water proton interfaces due to differences in precessional frequencies [13]. Cancer tissue gives low signal or appears isointense on T1-weighted images [18]. Cartilage invasion is therefore seen as loss of increased signal from the involved cartilage. However, early invasion by tumor would be difficult to detect if it were restricted to the low-signal rim.
Our investigation suggests that the conspicuity of cartilage invasion may be age-dependent and related to the degree of increased signal within the cartilage. The diagnostic accuracy of MRI may therefore be greater in older patients, who normally have higher signal within the laryngeal cartilages. We concentrated on the ability of MRI to show the normal signal patterns on T1-weighted images. Unenhanced images are thought to be most reliable for detecting cartilage invasion [2].

Contrast-enhanced studies have been performed to assess cartilage invasion [19]. They are more sensitive but with poor specificity. Abnormal enhancement may be related to tumor invasion, but reactive changes could result in similar appearances. The important observation is the absence of false negatives, which indicates that invasion can be excluded when normal signal intensity is seen within the cartilages [19].

Our investigation also underscores the importance of comparing the cartilage adjacent to tumor with the contralateral normal side. Heterogeneous signal adjacent to tumor should not be mistaken for neoplastic invasion if similar heterogeneous signal is present on the other side. An understanding of the expected signal within the laryngeal cartilages may help improve the diagnostic accuracy of MRI for detecting cartilage invasion by laryngeal carcinoma.

\section{References}

1. Muñoz A, Ramos A, Ferrando J, et al (1993) Laryngeal carcinoma: sclerotic appearance of the cricoid and arytenoid cartilage. CT-pathologic correlation. Radiology 189:433-437

2. Becker M, Zbaren P, Lang H, Stoupis C, Porcellini B, Vock P (1995) Neoplastic invasion of the laryngeal cartilage: comparison of MR imaging and CT with histopathologic correlation. Radiology 194: 661-669

3. Castelijns JA, Becker M, Hermans R (1996) Impact of cartilage invasion on treatment and prognosis of laryngeal cancer. Eur Radiol 6: 156-169
4. Zbaren P, Becker M, Lang H (1996) Pretherapeutic staging of laryngeal carcinoma. Clinical findings, computed tomography, and magnetic resonance imaging compared with histopathology. Cancer 77: 1263-1273

5. Becker M, Zbaren P, Delavelle J, et al (1997) Neoplastic invasion of the laryngeal cartilage: reassessment of criteria for diagnosis at CT. Radiology 203: $521-532$

6. Giron J, Joffre P, Serres-Cousine O, Senac JP (1993) CT and MR evaluation of laryngeal carcinomas. J Otolaryngol 22: $284-293$
7. Katsounakis J, Remy H, Vuong T, Gelinas M, Tabah R (1995) Impact of magnetic resonance imaging and computed tomography on the staging of laryngeal cancer. Eur Arch Otolaryngo 252: 206-208

8. Castelijns J, Gerritsen G, Kasier M, et al (1987) MRI of normal or cancerous cartilages: Histopathologic correlation. Laryngoscope 97: 1085-1093

9. Claassen H, Kirsch T, Simons G (1996) Cartilage canals in human thyroid cartilage characterized by immunolocalization of collagen types I, II, pro III, IV and X. Anat Embryol 94: 147-153 
10. Kikinis R, Wolfensberger M, Boesch C, Martin E (1989) Larynx: MR imaging at $2.35 \mathrm{~T}$. Radiology 171: 165-169

11. Castelijns JA, Doornbos J, Verbeeten B Jr, Vielvoye GJ, Bloem JL (1985) MR imaging of the normal larynx. J Comput Assist Tomogr 9: 919-925

12. McArdle CB, Bailey BJ, Amparo EG (1986) Surface coil magnetic resonance imaging of the normal larynx. Arch Otolaryngol Head Neck Surg 12: 616622
13. Sakai F, Gamsu G, Dillon WP, Kynch DA, Gilbert TJ (1990) MR imaging of the larynx at $1.5 \mathrm{~T}$. J Comput Assist Tomogr 14: 60-71

14. Hately W, Evison G, Samuel E (1965) The pattern of ossification in the laryngeal cartilages: a radiological study. Br J Radiol 38: 585-591

15. Keen JA, Wainwright J (1958) Ossification of the thyroid, cricoid and arytenoid cartilages. South Afr J Lab Clin Med 4: 83-108

16. Russo PE, Coin CG (1958) Calcification of the hyoid, thyroid, and tracheal cartilages in infancy: report of a case. Am J Roentgenol 80: 440-442

17. Yeager Vl, Lawson C, Archer C (1982) Ossification of the laryngeal cartilages as it relates to computed tomography. Invest Radiol 17: 11-19
18. Castelijns JA, Gerrtisen GJ, Kaiser MC, et al (1988) Invasion of laryngeal cartilage by cancer: comparison of CT and MR imaging. Radiology 167: 199206

19. Declercq A, Van den Hauwe L, Van Marck E, Van de Heyning PH, Spanoghe M, De Schepper AM (1998) Patterns of framework invasion in patients with laryngeal cancer: correlation of in vitro magnetic resonance imaging and pathologic findings. Acta Otolaryngol 118: $892-895$ 